\title{
Seletividade de Herbicidas Pré-emergentes para Gramíneas FORRAGEIRAS TROPICAIS ${ }^{1}$
}

\author{
Selectivity of Pre-Emergence Herbicides to Tropical Grass Forage
}

ALVES, E. ${ }^{2}$, MARTINS, D. ${ }^{3}$ e SOUZA, F.H.D. ${ }^{4}$

\begin{abstract}
RESUMO - Este trabalho constou de quatro estudos que foram realizados em casa de vegetação, nos quais se avaliou a seletividade de diferentes herbicidas, aplicados em pré-emergência, sobre algumas gramíneas forrageiras tropicais: Brachiaria decumbens, Brachiaria brizantha cv. Marandu e Panicum maximum cultivares Tanzânia e Mombaça. Os herbicidas e as doses utilizadas, em $\mathrm{g} \mathrm{ha}^{-1}$, para cada estudo foram: alachlor - 1.680 e 3.360, metolachlor - $1.200 \mathrm{e}$ 2.400 , diuron - 800 e 1.600 , imazaquin - 75 e 150, imazapyr - 250 e 500 , imazethapyr - 50 e 100 , clomazone - 450 e 900 , flumetsulam - 70 e 140 , ametryn - 625 e 1.250 , metribuzin - 525 e 1.050 e trifluralin - 900 e 1.800 , além de uma testemunha sem aplicação de herbicidas. Utilizou-se o delineamento experimental inteiramente casualizado, com seis repetições. $\mathrm{O}$ consumo de calda de aplicação foi de $180 \mathrm{~L} \mathrm{ha}^{-1}$, e a barra de aplicação continha quatro bicos de jato plano tipo 'Teejet' 110.02 , espaçados de $0,50 \mathrm{~m}$. Avaliou-se visualmente a intoxicação das plantas através de uma escala percentual de notas e, no final dos estudos, a altura e o peso de matéria seca de plantas. Para P. maximum cv. Mombaça, apenas os herbicidas imazaquin (75 $\mathrm{g} \mathrm{ha}^{-1}$ ), imazethapyr e flumetsulam, em ambas as doses testadas, foram seletivos. Para P. maximum cv. Tanzânia, nenhum dos herbicidas testados foi totalmente seletivo. Em relação a $B$. decumbens, os herbicidas imazaquin e imazethapyr, em ambas as doses, e ametryn (625 $\mathrm{g} \mathrm{ha}^{-1}$ ) foram seletivos. No caso de B. brizantha, os herbicidas diuron (800 $\left.\mathrm{g} \mathrm{ha}^{-1}\right)$, ametryn, imazaquin, imazethapyr e flumetsulam, em ambas as doses, apresentaram-se seletivos.
\end{abstract}

Palavras-chave: Brachiaria decumbens, Brachiaria brizantha, Panicum maximum cv. Tanzânia, Panicum maximum cv. Mombaça.

\begin{abstract}
This research comprised four experiments carried out under greenhouse conditions to evaluate the selectivity of herbicides, applied in pre-emergence conditions, on the following tropical grass forages: Brachiaria decumbens, Brachiaria brizantha $\mathrm{cv}$. Marandu, Panicum maximum cv. Tanzânia and Mombaça. The herbicides and rates $\left(g \mathrm{ha}^{-1}\right)$ applied for each experiment were alachlor (1.680 and 3,360), metolachlor (1,200 and 2,400), diuron (800 and 1,600), imazaquin (75 and 150), imazapyr (250 and 500), imazethapyr (50 and 100), clomazone (450 and 900), flumetsulam (70 and 140), ametryn (625 and 1,250), metribuzin (525 and 1,050), trifluralin $(900$ and 1,800), and a control plot, without herbicide aplication. The experimental design was completely randomized, with six replications. The spray volume was $180 \mathrm{~L} \mathrm{ha}^{-1}$ by four flat fan spray (TEEJET 110.02). Plant intoxication was evaluated by visual grading. At the end of the study plant height and dry matter weight were evaluated. For P. maximum cv. Mombaça, only imazaquin (75 $\left.\mathrm{g} \mathrm{ha}^{-1}\right)$, imazethapyr and flumetsulam at both rates tested were selective. For P. maximum cv. Tanzania, none of the tested herbicides were selective. Regardeless of the rate, imazaquin, imazethapyr and ametryn $\left(625 \mathrm{~g} \mathrm{ha}^{-1}\right)$ were selective to Brachiaria decumbens. For Brachiaria brizantha, the herbicides diuron $\left(800 \mathrm{~g} \mathrm{ha}^{-1}\right)$, ametryn, imazaquin, imazethapyr and flumetsulam at both tested rates were selective.
\end{abstract}

Key words: Brachiaria decumbens, Brachiaria brizantha, Panicum maximum cv. Tanzânia, Panicum maximum cv. Mombaça.

Recebido para publicação em 22/10/2001 e na forma revisada em 9/12/2002.

2 Aluno do programa de Pós-Graduação em Agronomia, Dep. de Produção Vegetal da Faculdade de Ciências Agrárias da Universidade Estadual Paulista - FCA/UNESP, Caixa Postal 237, 18603-970 Botucatu-SP; ${ }^{3}$ Professor Assistente Doutor, Dep. de Produção Vegetal - FCA/UNESP. ${ }^{4}$ Pesquisador Doutor da EMBRAPA/CPPSE, Caixa Postal 339, 13560-970 São Carlos-SP.

Planta Daninha, Viçosa-MG, v.20, n.3, p.457-464, 2002 


\section{INTRODUÇÃO}

A expansão de áreas cobertas por pastagens cultivadas é condicionada, normalmente, pela disponibilidade de sementes das plantas forrageiras. A área coberta por pastagens no Brasil é estimada em 177,7 milhões de hectares, sendo a área com pastagens cultivadas estimada em 99,7 milhões de hectares, representada na sua quase totalidade por gramineas.

Vários novos herbicidas foram introduzidos no mercado nacional, aumentando a possibilidade da eliminação seletiva de plantas indesejáveis. No entanto, possiveis efeitos de fitointoxicação desses produtos ainda não foram avaliados para as gramíneas forrageiras tropicais de interesse comercial no Brasil, o que limita seu uso nos campos de produção de sementes e em áreas de pastagens.

O controle das plantas daninhas é um problema com que todo pecuarista se depara constantemente. A invasão das pastagens está ligada à grande agressividade que essas plantas apresentam, aliada à degradação destas por manejo ou implantação inicial inadequada. Os principais problemas causados pelas plantas daninhas em pastagens são, segundo Belotto (1997), a competição direta por espaço, luz, água e nutrientes, bem como outros problemas indiretos, como meio de refúgio para animais peçonhentos.

As plantas daninhas, por serem agressivas e uma vez instaladas nos espaços abertos das pastagens, passam a competir diretamente pelos recursos do meio; para cada quilograma de planta daninha que se desenvolve, a redução da produção da pastagem toma proporção semelhante (Dias Filho, 1990). Segundo esse pesquisador, o aparente sucesso de muitas plantas daninhas na pastagem está mais diretamente ligado à capacidade que elas têm de se adaptar às práticas comuns no ambiente de pastagens, como a queima, o pastejo e a roçagem. Nesse grupo destacam-se o capim rabo-de-burro (Andropogom bicornis) e o capimnavalha (Paspalum virgatum).

O princípio básico do manejo da planta daninha é a prevenção de sua multiplicação. Shetty (1979) afirmou que em qualquer programa de controle de plantas daninhas deve ser levado em consideração o modo de reprodução e de dispersão das espécies que se deseja controlar. De acordo com Dias Filho (1990), quanto maior for a semelhança de uma planta invasora com a pastagem, maior será a dificuldade para controlar essa planta sem, também, prejudicar a cultura. O capim rabo-de-burro (A. bicornis) e o capim-navalha ( $P$. virgatum), por apresentarem muitas semelhanças com os capins utilizados nas pastagens, dificultam o uso de herbicidas e outras práticas de controle. A prevenção de plantas invasoras engloba todas as estratégias de manejo que impedem a entrada e o estabelecimento de novas plantas daninhas em uma determinada área.

As poucas pesquisas relativas ao uso de herbicidas na produção de sementes de pastagens tropicais buscam, prioritariamente, identificar, entre os produtos já liberados comercialmente, aqueles que apresentam potencial de uso entre as forrageiras (Loch \& Harvey, 1997). Essa linha de trabalho foi explorada por Veenstra \& Boonman (1974) na África, os quais avaliaram os efeitos de 23 herbicidas sobre várias gramíneas forrageiras (Brachiaria ruziziensis R. Germ. \& Evrard, Setaria sphacelata (Lam) Beauv var. sericea e Panicum spp.), bem como sobre algumas das principais plantas daninhas do Guênia. Considerando que dentro da família das gramíneas encontra-se amplo espectro de tolerância a herbicidas, faz-se necessário conhecer o comportamento das espécies e dos cultivares diante de cada um dos produtos potenciais.

Loch \& Harvey (1993) e Loch \& Harvey (1997) verificaram, em estudos realizados na Austrália, que, dos vários herbicidas testados, apenas atrazine, simazine, metribuzin, metsulfuron-methyl e chlorsulfuron mostraram-se promissores quanto à seletividade para gramíneas forrageiras. Convém ressaltar que nenhum destes estudos avaliou o grau de seletividade de vários herbicidas lançados e, tampouco, contemplou espécies e variedades de interesse no Brasil.

Nesse contexto, este trabalho foi proposto com o objetivo de avaliar a seletividade de herbicidas, em aplicações de pré-emergência, a algumas gramíneas forrageiras tropicais em utilização comercial no Brasil: Brachiaria decumbens Stapf cv. Basilisk, 
Brachiaria brizantha (Hochst) Stapf cv. Marandu e Panicum maximum Jacq. cv. Tanzânia e Panicum maximum Jacq. cv. Mombaça, com a finalidade de controle de plantas daninhas em áreas de pastagens e campos de produção de sementes.

\section{MATERIAL E MÉTODOS}

O estudo foi dividido em quatro experimentos, sendo um para cada espécie, instalados e conduzidos em casa de vegetação, no Núcleo de Pesquisas Avançadas em Matologia (NUPAM) da Faculdade de Ciências Agronômicas/ UNESP, Campus de Botucatu-SP, no período de dezembro de 1999 a junho de 2000.

As plantas foram cultivadas a partir de sementes certificadas, procedentes da EMBRAPA - Gado de Corte, sendo semeadas a $1 \mathrm{~cm}$ de profundidade em vasos plásticos com capacidade para $2 \mathrm{~kg}$ de solo seco. Foram realizados testes de germinação (Brasil, 1992) para determinar a quantidade de sementes viáveis necessária para se obter densidade final de 25 plantas por vaso. A umidade do solo, no decorrer do experimento, foi mantida por meio de regas, sendo realizadas diariamente ou conforme se observava visualmente a necessidade. A terra utilizada nos experimentos foi retirada de um Latossolo Roxo Eutrófico, de textura argilosa.

Os herbicidas e as doses, em $g$ ha $^{-1}$, utilizados nos estudos com Panicum maximum Tanzânia e Mombaça foram: metolachlor (2.400 e 1.200), diuron (1.600 e 800), imazaquin ( 150 e 75), imazapyr (500 e 250), imazethapyr (100 e 50), clomazone (900 e 450), flumetsulam (140 e 70), ametryn (1.250 e 625), metribuzin (1.050 e 525) e trifluralin (1.800 e 900). Para $B$. brizantha e $B$. decumbens foram testados os mesmos herbicidas, com a inclusão do alachlor (3.360 e $1.680 \mathrm{~g} \mathrm{ha}^{-1}$ ).

A pulverização foi realizada com um simulador de pulverização automatizado, regulado na freqüência de $45 \mathrm{~Hz}$, que correspondeu à velocidade de $1 \mathrm{~m} \mathrm{~s}^{-1}$. A temperatura e a umidade relativa do ar na sala de aplicação no momento da pulverização para $B$. decumbens e B. brizantha eram de $25{ }^{\circ} \mathrm{C}$ e $60 \%$, e para $P$. maximum, de $23^{\circ} \mathrm{C}$ e $75 \%$, respectivamente. A barra de aplicação continha quatro bicos de jato plano, tipo 'Teejet' 110.02 , espaçados de $0,50 \mathrm{~m}$ e posicionados a $0,50 \mathrm{~m}$ de altura dos vasos; utilizou-se um volume de calda de $180 \mathrm{~L} \mathrm{ha}^{-1}$.

O efeito dos tratamentos foi avaliado de acordo com escala percentual e visual de notas de 0 a 100, em que 0 foi considerado ausência de injúria e 100 a morte de plantas. As avaliações foram feitas em intervalos de no máximo sete dias a partir da aplicação. Por ocasião da finalização do período de estudos, aos 28 dias após a aplicação, as plantas foram avaliadas quanto à altura $(\mathrm{cm})$, sendo adotada a altura média de dez plantas por vaso, mensuradas desde sua base rente ao solo até o ápice da última folha expandida. Logo após a avaliação da altura, as plantas foram cortadas rente ao solo e colocadas em estufa com circulação forçada de ar, a $65^{\circ} \mathrm{C}$, por um período de sete dias, para determinação do peso de material seco. O delineamento experimental adotado foi o inteiramente casualizado, com seis repetições. Os resultados foram submetidos à análise de variância pelo teste " $F$ ", e as médias dos tratamentos, comparadas pelo teste " $\mathrm{t}$ " a $5 \%$ de probabilidade.

\section{RESULTADOS E DISCUSSÃO}

Nas Tabelas 1, 2, 3 e 4 estão apresentados os efeitos da aplicação dos diferentes herbicidas, pulverizados em pré-emergência, avaliados através da porcentagem de fitointoxicação em diferentes períodos de observação, da altura de plantas e do acúmulo de matéria seca.

Desde o início das avaliações, aos sete dias (7 DAA), e por ocasião do encerramento do estudo com P. maximum cv. Mombaça, aos 28 DAA, verificou-se que os herbicidas metolachlor, diuron, imazapyr, clomazone, metribuzin e trifluralin, nas duas doses testadas, e ametryn, na dose de $1.250 \mathrm{~g} \mathrm{ha}^{-1}$, não foram visualmente seletivos, pois proporcionaram injúrias severas às plantas, tanto que aos 28 DAA estas variaram de 75,6 a $100 \%$ (Tabela 1). Observaram-se, ainda, reduções severas na altura de plantas e no acúmulo de matéria seca. Victória Filho \& Lima (1999), ao estudarem os herbicidas metolachlor, diuron, clomazone e trifluralin, também observaram resultados semelhantes com relação à cultivar Mombaça. 
Tabela 1 - Seletividade de herbicidas aplicados em pré-emergência sobre plantas de Panicum maximum cv. Mombaça. Botucatu-SP, 2000

\begin{tabular}{|c|c|c|c|c|c|c|c|c|}
\hline \multirow{2}{*}{ Tratamento } & \multirow{2}{*}{$\mathrm{g} \mathrm{ha}^{-1}$} & \multicolumn{5}{|c|}{ Porcentagem de fitointoxicação (DAA) } & \multirow{2}{*}{$\begin{array}{c}\text { Altura de } \\
\text { plantas }(\mathrm{cm})\end{array}$} & \multirow{2}{*}{$\begin{array}{l}\text { Matéria sece } \\
\text { (g/vaso) }\end{array}$} \\
\hline & & 7 & 10 & 14 & 21 & 28 & & \\
\hline \multirow{2}{*}{ 1. metolachlor } & 2.400 & $80,0 \mathrm{ab}$ & $97,1 \mathrm{a}$ & $97,1 \mathrm{a}$ & $100,0 \mathrm{a}$ & $100,0 \mathrm{a}$ & $0,0 \mathrm{e}$ & $0,0 \mathrm{~g}$ \\
\hline & 1.200 & $76,6 \mathrm{ab}$ & $88,1 \mathrm{abc}$ & $88,1 \mathrm{~b}$ & $99,1 \mathrm{a}$ & $99,5 \mathrm{a}$ & $0,2 \mathrm{e}$ & $0,0 \mathrm{fg}$ \\
\hline \multirow{2}{*}{ 2. diuron } & 1.600 & $86,6 \mathrm{ab}$ & $96,1 \mathrm{ab}$ & $96,1 \mathrm{ab}$ & $99,5 \mathrm{a}$ & $99,6 \mathrm{a}$ & $2,0 \mathrm{de}$ & $0,0 \mathrm{~g}$ \\
\hline & 800 & $47,5 \mathrm{~d}$ & $58,8 \mathrm{~d}$ & $58,8 \mathrm{c}$ & $79,8 \mathrm{~b}$ & $75,6 \mathrm{c}$ & $11,2 \mathrm{~b}$ & 0,1 defg \\
\hline \multirow{2}{*}{ 3. imazaquin } & 150 & $18,3 \mathrm{ef}$ & $20,0 \mathrm{fg}$ & $30,8 \mathrm{~d}$ & $26,6 \mathrm{c}$ & 10,0 ef & $10,5 \mathrm{~b}$ & $0,2 \mathrm{bcd}$ \\
\hline & 75 & $7,8 \mathrm{fg}$ & $11,8 \mathrm{gh}$ & $14,3 \mathrm{f}$ & $6,6 \mathrm{e}$ & $0,0 \mathrm{f}$ & $13,1 \mathrm{ab}$ & $0,3 \mathrm{a}$ \\
\hline \multirow{2}{*}{ 4. imazapyr } & 500 & $38,3 \mathrm{~d}$ & $41,6 \mathrm{e}$ & $56,6 \mathrm{c}$ & $95,1 \mathrm{a}$ & $95,1 \mathrm{ab}$ & 3,5 cde & 0,1 efg \\
\hline & 250 & $20,8 \mathrm{e}$ & $25,0 \mathrm{f}$ & $25,0 \mathrm{de}$ & $78,3 \mathrm{~b}$ & $78,3 \mathrm{c}$ & $6,5 \mathrm{c}$ & 0,1 def \\
\hline \multirow{2}{*}{ 5. imazethapyr } & 100 & $0,3 \mathrm{~g}$ & $1,1 \mathrm{~h}$ & $1,1 \mathrm{~g}$ & $0,0 \mathrm{e}$ & $0,0 \mathrm{f}$ & $12,5 \mathrm{ab}$ & $0,3 \mathrm{a}$ \\
\hline & 50 & $1,8 \mathrm{~g}$ & $2,5 \mathrm{~h}$ & $2,5 \mathrm{~g}$ & $0,0 \mathrm{e}$ & $0,0 \mathrm{f}$ & $11,9 \mathrm{ab}$ & $0,2 \mathrm{abc}$ \\
\hline \multirow{2}{*}{ 6. clomazone } & 900 & $63,3 \mathrm{c}$ & $63,3 \mathrm{c}$ & $99,3 \mathrm{a}$ & $100,0 \mathrm{a}$ & $100,0 \mathrm{a}$ & $0,0 \mathrm{e}$ & $0,0 \mathrm{~g}$ \\
\hline & 450 & $78,3 \mathrm{ab}$ & $78,3 \mathrm{c}$ & $97,1 \mathrm{ab}$ & $97,8 \mathrm{a}$ & $98,3 \mathrm{ab}$ & $0,4 \mathrm{e}$ & $0,0 \mathrm{~g}$ \\
\hline \multirow{2}{*}{ 7. flumetsulam } & 140 & $1,6 \mathrm{~g}$ & $1,6 \mathrm{~h}$ & $3,5 \mathrm{~g}$ & $3,5 \mathrm{e}$ & $0,5 \mathrm{ef}$ & $13,5 \mathrm{ab}$ & $0,3 \mathrm{a}$ \\
\hline & 70 & $3,3 \mathrm{~g}$ & $3,6 \mathrm{~h}$ & $7,0 \mathrm{fg}$ & $4,3 \mathrm{e}$ & $1,0 \mathrm{ef}$ & $10,2 \mathrm{~b}$ & $0,2 \mathrm{abc}$ \\
\hline \multirow{2}{*}{ 8. ametryn } & 1.250 & 15,8 ef & $15,8 \mathrm{fg}$ & $29,1 \mathrm{~d}$ & $29,1 \mathrm{c}$ & $30,8 \mathrm{~d}$ & $11,6 \mathrm{ab}$ & 0,2 cde \\
\hline & 625 & $10,3 \mathrm{efg}$ & $10,3 \mathrm{gh}$ & $15,8 \mathrm{ef}$ & $15,8 \mathrm{~d}$ & $14,1 \mathrm{e}$ & $12,0 \mathrm{ab}$ & $0,2 \mathrm{abc}$ \\
\hline \multirow{2}{*}{ 9. metribuzin } & 1.050 & $72,5 \mathrm{bc}$ & $85,8 \mathrm{abc}$ & $97,1 \mathrm{ab}$ & $97,1 \mathrm{a}$ & $100,0 \mathrm{a}$ & $0,0 \mathrm{e}$ & $0,0 \mathrm{~g}$ \\
\hline & 525 & $75,0 \mathrm{~b}$ & $92,5 \mathrm{ab}$ & $94,0 \mathrm{ab}$ & $94,0 \mathrm{a}$ & $84,5 \mathrm{bc}$ & $4,7 \mathrm{~cd}$ & $0,0 \mathrm{fg}$ \\
\hline \multirow{2}{*}{ 10. trifluralin } & 1.800 & $82,5 a b$ & $90,8 \mathrm{ab}$ & $97,5 \mathrm{ab}$ & $97,5 \mathrm{a}$ & $100,0 \mathrm{a}$ & $0,0 \mathrm{e}$ & $0,0 \mathrm{~g}$ \\
\hline & 900 & $79,1 \mathrm{ab}$ & $85,0 \mathrm{bc}$ & $96,5 \mathrm{ab}$ & $96,5 \mathrm{a}$ & $100,0 \mathrm{a}$ & $0,0 \mathrm{e}$ & $0,0 \mathrm{~g}$ \\
\hline 11. testemunha & - & - & - & - & - & - & $15,2 \mathrm{a}$ & $0,3 \mathrm{ba}$ \\
\hline \multirow{3}{*}{\multicolumn{2}{|c|}{$\begin{array}{l}\text { F de tratamento } \\
\mathrm{CV}(\%) \\
\text { d.m.s. }\end{array}$}} & $66,49 * *$ & $89,26^{* *}$ & $120,00^{* *}$ & $192,92 * *$ & $81,07 * *$ & $20,16^{* *}$ & $10,63 * *$ \\
\hline & & 23,6 & 20,4 & 16,3 & 12,4 & 20,5 & 47,2 & 73,7 \\
\hline & & 11,6 & 11,3 & 10,3 & 8,7 & 13,9 & 3,59 & 0,10 \\
\hline
\end{tabular}

** Significativo a $1 \%$ de probabilidade.

Médias seguidas da mesma letra não diferem estatisticamente entre si pelo teste $\mathrm{t}(\mathrm{P}>0,05)$.

DAA - Dias Após a Aplicação.

Para os herbicidas imazaquin, na dose de $150 \mathrm{~g} \mathrm{ha}^{-1}$, e ametryn, na dose de $625 \mathrm{~g} \mathrm{ha}^{-1}$, as injúrias visuais observadas ao final do estudo não foram tão severas, porém, ao analisar os dados de altura de plantas e do acúmulo de matéria seca, notaram-se reduções médias para altura de planta de 31 e $22 \%$, respectivamente, e para o peso de matéria seca, de $34 \%$, para ambos os herbicidas.

Notou-se, também, que os herbicidas imazaquin, na dose de $75 \mathrm{~g} \mathrm{ha}^{-1}$, imazethapyr e flumetsulam, nas duas doses testadas, foram visualmente seletivos para a cultura de $P$. maximum cv. Mombaça. Esse fato pode ser confirmado pelos dados de altura de plantas e pelo acúmulo de matéria seca, para os quais não se observaram diferenças estatísticas em relação à testemunha. A menor dose de flumetsulam (70 $\mathrm{g} \mathrm{ha}^{-1}$ ) mostrou-se menos seletiva que a maior ( $140 \mathrm{~g} \mathrm{ha}^{-1}$ ); esse fato não se mostrou coerente com o comportamento esperado, apesar de não ocorrerem diferenças estatísticas entre as doses.

Ao final das avaliações para $P$. maximum cv. Tanzânia aos 28 DAA (Tabela 2), observouse que os herbicidas metolachlor, diuron, imazapyr, clomazone, metribuzin e trifluralin, nas duas doses testadas, não foram visualmente seletivos para as plantas, além de proporcionarem injúrias severas desde o início das avaliações, chegando a 100\%, o que levou plantas à morte. Verificaram-se, também, reduções drásticas na altura de plantas e no acúmulo de matéria seca, com exceção da altura de plantas na dose menor de diuron (800 $\mathrm{g} \mathrm{ha}^{-1}$ ), cujos dados não diferiram 
estatisticamente dos da testemunha. Contudo, deve-se destacar que, com redução de $71,7 \%$ no acúmulo de matéria seca, a cultura poderá chegar ao final de seu ciclo sem apresentar rendimento adequado.

Os herbicidas imazaquin (75 e $150 \mathrm{~g} \mathrm{ha}^{-1}$ ) e ametryn (1.250 $\mathrm{g} \mathrm{ha}^{-1}$ ) proporcionaram, ao final do estudo, injúrias visualmente severas às plantas do cultivar Tanzânia. Nos resultados de acúmulo de matéria seca notaram-se reduções médias de $67 \%$ para o imazaquin e de $65 \%$ para o ametryn. No entanto, ao analisar os resultados de altura de plantas, nota-se que não houve diferenças estatísticas, apesar de o herbicida imazaquin proporcionar $11 \%$ de redução com a dose de $150 \mathrm{~g} \mathrm{ha}^{-1}$ e $21 \%$ com a dose de $75 \mathrm{~g} \mathrm{ha}^{-1} \mathrm{e}$ o ametryn proporcionar redução de 5\% na altura de plantas.
Observou-se que os herbicidas ametryn (625 $\mathrm{g} \mathrm{ha}^{-1}$ ), imazethapyr e flumetsulam, nas duas doses testadas, foram visualmente seletivos para a cultura de $P$. maximum $\mathrm{cv}$. Tanzânia. Esse fato pode ser confirmado pelos dados de altura de plantas, que foram semelhantes aos da testemunha. Contudo, ao se observar o acúmulo de matéria seca, notamse efeitos deletérios na biomassa das plantas que receberam esses herbicidas nessas mesmas doses. Devido às contradições entre os resultados da avaliação de fitointoxicação e os da altura de plantas em relação ao acúmulo de matéria seca, a seletividade desses herbicidas deverá ser confirmada em novos estudos, inclusive no campo, onde seria avaliada a produtividade de sementes ao final do ciclo da cultura.

Tabela 2 - Seletividade de herbicidas aplicados em pré-emergência sobre plantas de Panicum maximum cv. Tanzânia. Botucatu-SP, 2000

\begin{tabular}{|c|c|c|c|c|c|c|c|c|}
\hline \multirow{2}{*}{ Tratamento } & \multirow{2}{*}{$\mathrm{g} \mathrm{ha}^{-1}$} & \multicolumn{5}{|c|}{ Porcentagem de fitointoxicação (DAA) } & \multirow{2}{*}{$\begin{array}{c}\text { Altura de } \\
\text { plantas }(\mathrm{cm})\end{array}$} & \multirow{2}{*}{$\begin{array}{c}\text { Matéria seca } \\
\text { (g/vaso) }\end{array}$} \\
\hline & & 7 & 10 & 14 & 21 & 28 & & \\
\hline \multirow{2}{*}{ 1. metolachlor } & 2.400 & $98,0 \mathrm{ab}$ & $100,0 \mathrm{a}$ & $100,0 \mathrm{a}$ & $100,0 \mathrm{a}$ & $100,0 \mathrm{a}$ & $0,0 \mathrm{e}$ & $0,0 \mathrm{~h}$ \\
\hline & 1.200 & $94,0 \mathrm{ab}$ & 98,6 a & $100,0 \mathrm{a}$ & $100,0 \mathrm{a}$ & $100,0 \mathrm{a}$ & $0,0 \mathrm{e}$ & $0,0 \mathrm{~h}$ \\
\hline \multirow{2}{*}{ 2. diuron } & 1.600 & $79,5 \mathrm{c}$ & $86,1 \mathrm{~b}$ & $90,5 \mathrm{a}$ & $92,3 \mathrm{a}$ & $94,8 \mathrm{a}$ & $2,5 \mathrm{de}$ & $0,02 \mathrm{~h}$ \\
\hline & 800 & $32,1 \mathrm{~d}$ & $47,1 \mathrm{c}$ & $55,5 \mathrm{c}$ & $62,3 \mathrm{c}$ & $68,6 \mathrm{~b}$ & $10,0 \mathrm{abc}$ & 0,13 efg \\
\hline \multirow{2}{*}{ 3. imazaquin } & 150 & $16,6 \mathrm{fg}$ & $27,5 \mathrm{~d}$ & $27,5 \mathrm{e}$ & $27,5 \mathrm{e}$ & $30,3 \mathrm{~d}$ & $8,9 \mathrm{c}$ & $0,13 \mathrm{ef}$ \\
\hline & 75 & $10,0 \mathrm{gh}$ & $11,3 \mathrm{e}$ & $13,0 \mathrm{f}$ & $15,8 \mathrm{ef}$ & $18,3 \mathrm{de}$ & $7,9 \mathrm{c}$ & $0,17 \mathrm{de}$ \\
\hline \multirow{2}{*}{ 4. imazapyr } & 500 & $25,8 \mathrm{de}$ & $25,8 \mathrm{~d}$ & "77,5 b & $98,6 \mathrm{a}$ & 99,0 a & $1,0 \mathrm{e}$ & $0,04 \mathrm{gh}$ \\
\hline & 250 & 20,0 ef & $25,0 \mathrm{~d}$ & $55,0 \mathrm{c}$ & $80,0 \mathrm{~b}$ & $80,0 \mathrm{~b}$ & $3,5 \mathrm{~d}$ & $0,07 \mathrm{fgh}$ \\
\hline \multirow{2}{*}{ 5. imazethapyr } & 100 & $0,5 \mathrm{i}$ & $2,0 \mathrm{e}$ & $1,1 \mathrm{f}$ & $0,0 \mathrm{~g}$ & $0,0 \mathrm{f}$ & $12,4 \mathrm{a}$ & $0,3 \mathrm{bc}$ \\
\hline & 50 & $0,6 \mathrm{i}$ & $2,6 \mathrm{e}$ & $1,8 \mathrm{f}$ & $0,0 \mathrm{~g}$ & $0,0 \mathrm{f}$ & $11,5 \mathrm{ab}$ & $0,32 \mathrm{~b}$ \\
\hline \multirow{2}{*}{ 6. clomazone } & 900 & $99,0 \mathrm{a}$ & $99,3 \mathrm{a}$ & $99,3 \mathrm{a}$ & $100,0 \mathrm{a}$ & $100,0 \mathrm{a}$ & $0,0 \mathrm{e}$ & $0,0 \mathrm{~h}$ \\
\hline & 450 & $93,1 \mathrm{ab}$ & $97,6 \mathrm{a}$ & 97,6 a & $99,1 \mathrm{a}$ & $100,0 \mathrm{a}$ & $0,0 \mathrm{e}$ & $0,0 \mathrm{~h}$ \\
\hline \multirow{2}{*}{ 7. flumetsulam } & 140 & $1,0 \mathrm{i}$ & $2,8 \mathrm{e}$ & $2,8 \mathrm{f}$ & $3,0 \mathrm{~g}$ & $3,0 \mathrm{f}$ & $11,5 \mathrm{ab}$ & $0,3 \mathrm{bc}$ \\
\hline & 70 & 8,0 ghi & $8,0 \mathrm{e}$ & $2,8 \mathrm{f}$ & $1,6 \mathrm{~g}$ & $1,0 \mathrm{f}$ & $10,1 \mathrm{abc}$ & 0,22 cde \\
\hline \multirow{2}{*}{ 8. ametryn } & 1.250 & 23,3 def & $35,8 \mathrm{~d}$ & $42,5 \mathrm{~d}$ & $48,3 \mathrm{~d}$ & $48,5 \mathrm{c}$ & $9,5 \mathrm{bc}$ & $0,16 \mathrm{e}$ \\
\hline & 625 & 2,3 hi & $5,3 \mathrm{e}$ & $7,0 \mathrm{f}$ & $8,3 \mathrm{fg}$ & $5,6 \mathrm{ef}$ & $9,5 \mathrm{bc}$ & $0,26 \mathrm{bcd}$ \\
\hline \multirow{2}{*}{ 9. metribuzin } & 1.050 & $89,1 \mathrm{~b}$ & $98,5 \mathrm{a}$ & $100,0 \mathrm{a}$ & $100,0 \mathrm{a}$ & $100,0 \mathrm{a}$ & $0,0 \mathrm{e}$ & $0,0 \mathrm{~h}$ \\
\hline & 525 & $94,6 \mathrm{ab}$ & $96,0 \mathrm{ab}$ & $96,0 \mathrm{a}$ & $97,3 \mathrm{a}$ & $98,3 \mathrm{a}$ & $4,9 \mathrm{~d}$ & $0,03 \mathrm{~h}$ \\
\hline \multirow{2}{*}{ 10. trifluralin } & 1.800 & $93,1 \mathrm{ab}$ & $97,0 \mathrm{ab}$ & $97,0 \mathrm{a}$ & $97,0 \mathrm{a}$ & $100,0 \mathrm{a}$ & $0,0 \mathrm{e}$ & $0,0 \mathrm{~h}$ \\
\hline & 900 & $89,1 \mathrm{~b}$ & $97,0 \mathrm{ab}$ & $97,0 \mathrm{a}$ & $97,0 \mathrm{a}$ & $100,0 \mathrm{a}$ & $0,0 \mathrm{e}$ & $0,0 \mathrm{~h}$ \\
\hline 11. testemunha & - & - & - & - & - & - & $10,0 \mathrm{abc}$ & $0,46 \mathrm{a}$ \\
\hline \multirow{3}{*}{\multicolumn{2}{|c|}{$\begin{array}{l}\text { F de tratamento } \\
\mathrm{CV}(\%) \\
\text { d.m.s. }\end{array}$}} & $163,63 * *$ & $112,83 * *$ & $87,73 * *$ & $98,59 * *$ & $88,94 * *$ & $18,72 * *$ & $28,78 * *$ \\
\hline & & 16,3 & 18,2 & 18,6 & 17,1 & 17,9 & 62,2 & 41,2 \\
\hline & & 9,1 & 11,0 & 12,4 & 12,0 & 12,8 & 0,09 & 2,55 \\
\hline
\end{tabular}

** Significativo a $1 \%$ de probabilidade.

Médias seguidas da mesma letra não diferem estatisticamente entre si pelo teste $\mathrm{t}(\mathrm{P}>0,05)$.

DAA - Dias Após a Aplicação. 
Tabela 3 - Seletividade de herbicidas aplicados em pré-emergência sobre plantas de Brachiaria brizantha cv. Marandu. Botucatu-SP, 2000

\begin{tabular}{|c|c|c|c|c|c|c|c|c|}
\hline \multirow{2}{*}{ Tratamento } & \multirow{2}{*}{$\mathrm{g} \mathrm{ha}^{-1}$} & \multicolumn{5}{|c|}{ Porcentagem de fitointoxicação (DAA) } & \multirow{2}{*}{$\begin{array}{c}\text { Altura de } \\
\text { plantas }(\mathrm{cm})\end{array}$} & \multirow{2}{*}{$\begin{array}{c}\text { Matéria seca } \\
\text { (g/vaso) }\end{array}$} \\
\hline & & 7 & 10 & 14 & 21 & 28 & & \\
\hline \multirow{2}{*}{ 1. alachlor } & 3.360 & $96,8 \mathrm{a}$ & 98,0 a & $98,1 \mathrm{a}$ & $100,0 \mathrm{a}$ & $100,0 \mathrm{a}$ & $0,0 \mathrm{~h}$ & $0,0 \mathrm{~g}$ \\
\hline & 1.680 & $98,0 \mathrm{a}$ & $97,8 \mathrm{a}$ & $97,3 \mathrm{a}$ & $100,0 \mathrm{a}$ & $100,0 \mathrm{a}$ & $0,0 \mathrm{~h}$ & $0,0 \mathrm{~g}$ \\
\hline \multirow{2}{*}{ 2. metolachlor } & 2.400 & $97,5 \mathrm{a}$ & $97,3 \mathrm{a}$ & $96,5 \mathrm{a}$ & $98,8 \mathrm{a}$ & $98,3 \mathrm{a}$ & $0,0 \mathrm{~h}$ & $0,0 \mathrm{~g}$ \\
\hline & 1.200 & $97,5 \mathrm{a}$ & $95,3 \mathrm{a}$ & $94,3 \mathrm{a}$ & $96,3 \mathrm{a}$ & $96,3 \mathrm{a}$ & $0,0 \mathrm{~h}$ & $0,0 \mathrm{~g}$ \\
\hline \multirow{2}{*}{ 3. diuron } & 1.600 & $10,5 \mathrm{~d}$ & $22,5 \mathrm{~d}$ & $50,8 \mathrm{~b}$ & $60,8 \mathrm{c}$ & $60,8 \mathrm{c}$ & $10,0 \mathrm{de}$ & $0,2 \mathrm{e}$ \\
\hline & 800 & $0,0 \mathrm{f}$ & $0,0 \mathrm{f}$ & $0,0 \mathrm{~d}$ & $0,0 \mathrm{~d}$ & $0,0 \mathrm{~d}$ & $11,2 \mathrm{~cd}$ & $0,6 \mathrm{bc}$ \\
\hline \multirow{2}{*}{ 4. imazaquin } & 150 & $0,0 \mathrm{f}$ & $0,0 \mathrm{f}$ & $0,0 \mathrm{~d}$ & $0,0 \mathrm{~d}$ & $0,0 \mathrm{~d}$ & $12,0 \mathrm{bc}$ & $0,7 \mathrm{a}$ \\
\hline & 75 & $0,8 \mathrm{f}$ & $0,5 \mathrm{f}$ & $0,5 \mathrm{~d}$ & $0,8 \mathrm{~d}$ & $0,8 \mathrm{~d}$ & $12,0 \mathrm{bc}$ & $0,7 \mathrm{a}$ \\
\hline \multirow{2}{*}{ 5. imazapyr } & 500 & $28,1 \mathrm{c}$ & $38,3 \mathrm{c}$ & $60,8 \mathrm{~b}$ & $73,8 \mathrm{~b}$ & $73,8 \mathrm{~b}$ & $4,9 \mathrm{~g}$ & $0,3 \mathrm{e}$ \\
\hline & 250 & $6,3 \mathrm{de}$ & $15,0 \mathrm{e}$ & $31,6 \mathrm{c}$ & $53,3 \mathrm{c}$ & $53,3 \mathrm{c}$ & $8,8 \mathrm{e}$ & $0,5 \mathrm{~d}$ \\
\hline \multirow{2}{*}{ 6. imazethapyr } & 100 & $0,0 \mathrm{f}$ & $0,0 \mathrm{f}$ & $0,0 \mathrm{~d}$ & $0,0 \mathrm{~d}$ & $0,0 \mathrm{~d}$ & $10,7 \mathrm{~cd}$ & $0,6 \mathrm{ab}$ \\
\hline & 50 & $0,0 \mathrm{f}$ & $0,0 \mathrm{f}$ & $0,0 \mathrm{~d}$ & $0,0 \mathrm{~d}$ & $0,0 \mathrm{~d}$ & $12,9 \mathrm{~b}$ & $0,7 \mathrm{a}$ \\
\hline \multirow{2}{*}{ 7. clomazone } & 900 & $100,0 \mathrm{a}$ & $100,0 \mathrm{a}$ & $100,0 \mathrm{a}$ & $100,0 \mathrm{a}$ & $100,0 \mathrm{a}$ & $0,0 \mathrm{~h}$ & $0,0 \mathrm{~g}$ \\
\hline & 450 & $98,1 \mathrm{a}$ & $99,0 \mathrm{a}$ & $100,0 \mathrm{a}$ & $100,0 \mathrm{a}$ & $100,0 \mathrm{a}$ & $0,4 \mathrm{~h}$ & $0,0 \mathrm{fg}$ \\
\hline \multirow{2}{*}{ 8. flumetsulam } & 140 & $0,0 \mathrm{f}$ & $0,0 \mathrm{f}$ & $1,8 \mathrm{~d}$ & $0,0 \mathrm{~d}$ & $0,0 \mathrm{~d}$ & $11,3 \mathrm{~cd}$ & $0,6 \mathrm{abc}$ \\
\hline & 70 & $0,0 \mathrm{f}$ & $0,0 \mathrm{f}$ & $0,0 \mathrm{~d}$ & $0,0 \mathrm{~d}$ & $0,0 \mathrm{~d}$ & $10,5 \mathrm{~d}$ & $0,7 \mathrm{a}$ \\
\hline \multirow{2}{*}{ 9. ametryn } & 1.250 & $3,0 \mathrm{f}$ & $0,5 \mathrm{f}$ & $1,8 \mathrm{~d}$ & $1,1 \mathrm{~d}$ & $1,6 \mathrm{~d}$ & $13,1 \mathrm{ab}$ & $0,5 \mathrm{~cd}$ \\
\hline & 625 & $0,0 \mathrm{f}$ & $0,0 \mathrm{f}$ & $0,0 \mathrm{~d}$ & $0,0 \mathrm{~d}$ & $0,0 \mathrm{~d}$ & $11,1 \mathrm{~cd}$ & $0,6 \mathrm{ab}$ \\
\hline \multirow{2}{*}{ 10. metribuzin } & 1.050 & $67,5 \mathrm{~b}$ & $99,6 \mathrm{a}$ & $100,0 \mathrm{a}$ & $100,0 \mathrm{a}$ & $100,0 \mathrm{a}$ & $0,0 \mathrm{~h}$ & $0,0 \mathrm{~g}$ \\
\hline & 525 & $26,6 \mathrm{c}$ & $82,1 \mathrm{~b}$ & $90,3 \mathrm{a}$ & $94,1 \mathrm{a}$ & $98,3 \mathrm{a}$ & $7,0 \mathrm{f}$ & $0,1 \mathrm{f}$ \\
\hline \multirow{2}{*}{ 11. trifluralin } & 1.800 & $100,0 \mathrm{a}$ & $100,0 \mathrm{a}$ & $100,0 \mathrm{a}$ & $100,0 \mathrm{a}$ & $100,0 \mathrm{a}$ & $0,0 \mathrm{~h}$ & $0,0 \mathrm{~g}$ \\
\hline & 900 & $100,0 \mathrm{a}$ & $100,0 \mathrm{a}$ & $100,0 \mathrm{a}$ & $100,0 \mathrm{a}$ & $100,0 \mathrm{a}$ & $0,8 \mathrm{~h}$ & $0,0 \mathrm{~g}$ \\
\hline 12. testemunha & - & - & - & - & - & - & $14,5 \mathrm{a}$ & $0,6 \mathrm{bcd}$ \\
\hline \multirow{3}{*}{\multicolumn{2}{|c|}{$\begin{array}{l}\text { F de tratamento } \\
\mathrm{CV}(\%) \\
\text { d.m.s. }\end{array}$}} & $683,50 * *$ & $401,52 * *$ & $122,48^{* *}$ & $222,16^{* *}$ & $245,00 * *$ & $128,08^{* *}$ & $69,95 * *$ \\
\hline & & 10,1 & 12,1 & 20,1 & 14,5 & 13,7 & 18,4 & 27,3 \\
\hline & & 4,9 & 6,5 & 11,7 & 8,8 & 8,4 & 1,3 & 0,10 \\
\hline
\end{tabular}

** - Significativo a $1 \%$ de probabilidade.

Médias seguidas da mesma letra não diferem estatisticamente entre si pelo teste $t(P>0,05)$.

DAA - Dias Após a Aplicação.

Ao final do estudo com Brachiaria brizantha cv. Marandu (Tabela 3), observou-se que os herbicidas alachlor, metolachlor, imazapyr, clomazone, metribuzin e trifluralin, nas doses testadas, e diuron a $1.600 \mathrm{~g} \mathrm{ha}^{-1}$ não foram visualmente seletivos para as plantas de $B$. brizantha, pois proporcionaram injúrias severas às plantas desde o início das avaliações. Ao final do estudo, as injúrias decorrentes dos herbicidas variaram de 53,3 a 100\%; com exceção do imazapyr nas duas doses testadas e do metribuzin na dose de $525 \mathrm{~g} \mathrm{ha}^{-1}$, todos os demais herbicidas estudados levaram à morte completa das plantas, o que pode ser confirmado pelo acúmulo de matéria seca.

Os herbicidas diuron ( $800 \mathrm{~g} \mathrm{ha}^{-1}$ ), ametryn, imazaquin, imazethapyr e flumetsulam, nas doses testadas, apresentaram-se visualmente seletivos para a cultura de $B$. brizantha cv. Marandu, o que pode ser confirmado pelos dados de acúmulo de matéria seca, que foram semelhantes aos da testemunha. Possiveis reduções de produtividade da cultura, decorrentes da redução na altura de plantas proporcionada por esses herbicidas que se apresentaram visualmente seletivos, somente poderão ser confirmadas em estudos nos quais a cultura seja conduzida até o final do ciclo, uma vez que não houve efeito sobre o acúmulo de matéria seca.

Por ocasião do encerramento do estudo (28 DAA) com as plantas de $B$. decumbens cv. Basilisk (Tabela 4), verificou-se que os herbicidas alachlor, metolachlor, clomazone, 
metribuzin e trifluralin, nas doses testadas, diuron a $1.600 \mathrm{~g} \mathrm{ha}^{-1} \mathrm{e}$ imazapyr a $500 \mathrm{~g} \mathrm{ha}^{-1}$ não foram visualmente seletivos para as plantas de $B$. decumbens cv. Basilisk, pois proporcionaram injúrias severas desde o início do estudo, atingindo de 98 a 100\% aos 28 DAA. Observaram-se, também, reduções drásticas na altura de plantas e no acúmulo de matéria seca, chegando, em alguns casos, à morte das plantas. Esses resultados confirmam os de Victória Filho \& Lima (1999), que avaliaram os herbicidas metolachlor, diuron, clomazone e trifluralin, mas com a utilização de doses mais elevadas, e observaram a não-seletividade desses produtos.

O herbicida imazapyr na dose de $250 \mathrm{~g} \mathrm{ha}^{-1}$ inicialmente provocou leves injúrias, as quais foram se tornando mais acentuadas no decorrer do estudo, reduzindo a altura de plantas em $41 \%$ e o acúmulo de matéria seca em $25,9 \%$, o que pode caracterizar o herbicida como não-seletivo para a espécie em questão.

Para o herbicida flumetsulam na dose de $70 \mathrm{~g} \mathrm{ha}^{-1}$, as injúrias observadas ao final do estudo não foram tão severas. Entretanto, quando foi utilizada a dose de $140 \mathrm{~g} \mathrm{ha}^{-1}$, foram constatadas reduções da ordem de 39,2 e $25,9 \%$ para altura de plantas e peso de matéria seca, respectivamente, apesar de a última característica não apresentar diferença estatística em relação à testemunha. Os herbicidas diuron (800 $\mathrm{g} \mathrm{ha}^{-1}$ ), imazaquin, imazethapyr e ametryn, nas doses testadas, foram visualmente seletivos para a cultura de $B$. decumbens cv. Basilisk, podendo isso ser confirmado pelos dados de altura de plantas e pelo acúmulo de matéria seca, com valores semelhantes aos da testemunha.

Tabela 4 - Seletividade de herbicidas aplicados em pré-emergência sobre plantas de Brachiaria decumbens cv. Basilisk. Botucatu-SP, 2000

\begin{tabular}{|c|c|c|c|c|c|c|c|c|}
\hline \multirow{2}{*}{ Tratamento } & \multirow{2}{*}{$\mathrm{g} \mathrm{ha}^{-1}$} & \multicolumn{5}{|c|}{ Porcentagem de fitointoxicação (DAA) } & \multirow{2}{*}{$\begin{array}{c}\text { Altura de } \\
\text { plantas }(\mathrm{cm})\end{array}$} & \multirow{2}{*}{$\begin{array}{c}\text { Matéria seca } \\
\text { (g/vaso) }\end{array}$} \\
\hline & & 7 & 10 & 14 & 21 & 28 & & \\
\hline \multirow{2}{*}{ 1. alachlor } & 3.360 & $98,1 \mathrm{a}$ & $99,0 \mathrm{a}$ & $99,0 \mathrm{a}$ & $99,8 \mathrm{a}$ & $100,0 \mathrm{a}$ & $0,0 \mathrm{~h}$ & $0,0 \mathrm{~h}$ \\
\hline & 1.680 & $97,8 \mathrm{a}$ & $98,1 \mathrm{a}$ & $98,1 \mathrm{a}$ & $99,0 \mathrm{a}$ & $100,0 \mathrm{a}$ & $0,0 \mathrm{~h}$ & $0,0 \mathrm{~h}$ \\
\hline \multirow{2}{*}{ 2. metolachlor } & 2.400 & $97,3 \mathrm{a}$ & $98,0 \mathrm{a}$ & $98,0 \mathrm{a}$ & $98,6 \mathrm{a}$ & $99,8 \mathrm{a}$ & $0,0 \mathrm{~h}$ & $0,0 \mathrm{~h}$ \\
\hline & 1.200 & $96,5 \mathrm{a}$ & $96,5 \mathrm{a}$ & $96,5 \mathrm{a}$ & $97,5 \mathrm{a}$ & $99,1 \mathrm{a}$ & $0,0 \mathrm{~h}$ & $0,0 \mathrm{~h}$ \\
\hline \multirow{2}{*}{ 3. diuron } & 1.600 & $45,0 \mathrm{c}$ & $52,5 \mathrm{c}$ & $52,5 \mathrm{c}$ & $79,1 \mathrm{~b}$ & $86,8 \mathrm{~b}$ & 9,4 efd & $0,1 \mathrm{~g}$ \\
\hline & 800 & $0,0 \mathrm{e}$ & $0,0 \mathrm{~g}$ & $0,0 \mathrm{~g}$ & $0,0 \mathrm{~g}$ & $0,0 \mathrm{~g}$ & $11,1 \mathrm{bcd}$ & $0,5 \mathrm{bc}$ \\
\hline \multirow{2}{*}{ 4. imazaquin } & 150 & $0,0 \mathrm{e}$ & $0,0 \mathrm{~g}$ & $0,0 \mathrm{~g}$ & $0,0 \mathrm{~g}$ & $0,0 \mathrm{~g}$ & $13,5 \mathrm{~b}$ & $0,6 \mathrm{~b}$ \\
\hline & 75 & $0,0 \mathrm{e}$ & $0,0 \mathrm{~g}$ & $0,0 \mathrm{~g}$ & $0,0 \mathrm{~g}$ & $0,0 \mathrm{~g}$ & $16,6 a$ & $0,9 \mathrm{a}$ \\
\hline \multirow{2}{*}{ 5. imazapyr } & 500 & $30,5 \mathrm{~d}$ & $45,0 \mathrm{c}$ & $45,0 \mathrm{c}$ & $65,0 \mathrm{c}$ & $59,1 \mathrm{c}$ & $8,2 \mathrm{ef}$ & $0,3 \mathrm{f}$ \\
\hline & 250 & $3,8 \mathrm{e}$ & $22,5 \mathrm{~d}$ & $22,5 \mathrm{~d}$ & $25,0 \mathrm{e}$ & $40,0 \mathrm{~d}$ & $7,3 \mathrm{f}$ & 0,4 ef \\
\hline \multirow{2}{*}{ 6. imazethapyr } & 100 & $0,0 \mathrm{e}$ & $1,0 \mathrm{fg}$ & $1,0 \mathrm{fg}$ & $4,8 \mathrm{~g}$ & $8,5 \mathrm{fg}$ & $11,9 \mathrm{bcd}$ & 0,5 bcde \\
\hline & 50 & $0,0 \mathrm{e}$ & $0,0 \mathrm{~g}$ & $0,0 \mathrm{~g}$ & $1,1 \mathrm{~g}$ & $1,0 \mathrm{~g}$ & 10,2 cde & $0,5 \mathrm{bcd}$ \\
\hline \multirow{2}{*}{ 7. clomazone } & 900 & $100,0 \mathrm{a}$ & $100,0 \mathrm{a}$ & $100,0 \mathrm{a}$ & $100,0 \mathrm{a}$ & $100,0 \mathrm{a}$ & $0,0 \mathrm{~h}$ & $0,0 \mathrm{~h}$ \\
\hline & 450 & $98,3 \mathrm{a}$ & $99,6 \mathrm{a}$ & $99,6 \mathrm{a}$ & $99,8 \mathrm{a}$ & $100,0 \mathrm{a}$ & $0,0 \mathrm{~h}$ & $0,0 \mathrm{~h}$ \\
\hline \multirow{2}{*}{ 8. flumetsulam } & 140 & $1,0 \mathrm{e}$ & $15,0 \mathrm{de}$ & $15,0 \mathrm{de}$ & $46,6 \mathrm{~d}$ & $29,6 \mathrm{e}$ & 7,6 ef & 0,4 def \\
\hline & 70 & $0,0 \mathrm{e}$ & 10,0 ef & 10,0 ef & $15,0 \mathrm{f}$ & $10,0 \mathrm{f}$ & $11,1 \mathrm{bcd}$ & 0,4 cde \\
\hline \multirow{2}{*}{ 9. ametryn } & 1.250 & $1,3 \mathrm{e}$ & $0,0 \mathrm{~g}$ & $0,0 \mathrm{~g}$ & $2,1 \mathrm{~g}$ & $3,6 \mathrm{fg}$ & $13,1 \mathrm{~b}$ & $0,3 \mathrm{f}$ \\
\hline & 625 & $0,0 \mathrm{e}$ & $0,0 \mathrm{~g}$ & $0,0 \mathrm{~g}$ & $0,0 \mathrm{~g}$ & $0,3 \mathrm{~g}$ & $11,5 \mathrm{bcd}$ & $0,6 \mathrm{~b}$ \\
\hline \multirow{2}{*}{ 10. metribuzin } & 1.050 & $69,1 \mathrm{~b}$ & $86,5 \mathrm{~b}$ & $86,5 \mathrm{~b}$ & $95,5 \mathrm{a}$ & $98,0 \mathrm{a}$ & $0,0 \mathrm{~h}$ & $0,0 \mathrm{~h}$ \\
\hline & 525 & $63,3 \mathrm{~b}$ & 92,3 ba & $92,3 \mathrm{ba}$ & $98,5 \mathrm{a}$ & $98,3 \mathrm{a}$ & $0,0 \mathrm{~h}$ & $0,0 \mathrm{~h}$ \\
\hline \multirow{2}{*}{ 11. trifluralin } & 1.800 & $100,0 \mathrm{a}$ & $100,0 \mathrm{a}$ & $100,0 \mathrm{a}$ & $100,0 \mathrm{a}$ & $100,0 \mathrm{a}$ & $0,0 \mathrm{~h}$ & $0,0 \mathrm{~h}$ \\
\hline & 900 & $100,0 \mathrm{a}$ & $99,3 \mathrm{a}$ & $99,3 \mathrm{a}$ & $99,8 \mathrm{a}$ & $100,0 \mathrm{a}$ & $0,0 \mathrm{~h}$ & $0,0 \mathrm{~h}$ \\
\hline 12. testemunha & - & - & - & - & - & - & $12,5 \mathrm{bc}$ & $0,54 \mathrm{bcd}$ \\
\hline \multirow{3}{*}{\multicolumn{2}{|c|}{$\begin{array}{l}\text { F de tratamento } \\
\mathrm{CV}(\%) \\
\text { d.m.s. }\end{array}$}} & $143,61 * *$ & $169,59 * *$ & $169,59 * *$ & $173,08 * *$ & $205,44 * *$ & $35,89 * *$ & $56,88 * *$ \\
\hline & & 20,4 & 16,8 & 16,8 & 15,0 & 13,8 & 35,5 & 32,0 \\
\hline & & 10,6 & 9,7 & 9,7 & 9,6 & 8,9 & 2,6 & 0,1 \\
\hline
\end{tabular}

** Significativo a $1 \%$ de probabilidade.

Médias seguidas da mesma letra não diferem estatisticamente entre si pelo teste $t(P>0,05)$.

DAA - Dias Após a Aplicação. 
Com exceção da maior dose de ametryn (1.250 $\mathrm{g} \mathrm{ha}^{-1}$ ) - com a qual se observou reduções de $44 \%$ no acúmulo de matéria seca - e da altura de plantas, não foram observadas diferenças estatísticas em relação à testemunha. A não-seletividade observada na maior dose de ametryn está de acordo com os resultados obtidos por Victória Filho \& Lima (1999), que, utilizando doses superiores a $1.250 \mathrm{~g} \mathrm{ha}^{-1}$, também puderam observar a não-seletividade desse herbicida para a cultura de B. decumbens cv. Basilisk.

\section{LITERATURA CITADA}

BELOTTO, E. E. Controle de plantas daninhas em pastagens. In: SIMPÓSIO SOBRE HERBICIDAS E PLANTAS DANINHAS, 1., 1997, Dourados. Resumos... Dourados: s.n., 1997. p. 118-30.

BRASIL. Ministério da Agricultura. Regras para análise de sementes. Brasília: Departamento Nacional de Produção Vegetal, Divisão de Sementes e Mudas, 1992. 365 p.

DIAS FILHO, M. B. Plantas invasoras em pastagens cultivadas na Amazônia: Estratégias de manejo e controle. Brasília: Empresa Brasileira de Pesquisa Agropecuária, 1990. 103 p. (Documento, 52).
LOCH, D. S. L.; HARVEY, G. L. Developing herbicide strategies for tropical herbage seed crops. In: AUSTRALIAN NEW CROPS CONFERENCE, 1., 1996, Queensland. Proceedings... Queensland: Gaton College, 1997. p. 273-282.

LOCH, D. S. L.; HARVEY, G. L. Preliminary screening of 17 tropical grasses for tolerance to eight graminaceous herbicides. In: GRASSLAND CONGRESS, 17., 1993, New Zeland. Proceedings... Lincoln: New Zealand, 1993. p. 1646-1648.

SHETTY, S. V. R. Approaches to integrated weed management in maize and sorghum in tropical and subtropical areas. In: ASIAN PACIFIC WEED SCIENCE SOCIETY CONFERENCE, 7., 1979, Sydney.

Proceedings... Bathurst: Australian Weed Science Societies for Asian, 1979. p.87-93.

VEENSTRA, T.; BOONMAN, J. G. Chemical weed control in tropical grasses and legumes. In: EAST AFRICAN WEED CONTROL CONFERENCE, 5., 1974, Nairobi. Proceedings... Nairobi: [s.n.], 1974. p. 139-149.

VICTÓRIA FILHO, R.; LIMA, J. F. Controle do capimbraquiaria (Brachiaria decumbens Stapf) na implantação de algumas gramíneas forrageiras. In: CONGRESO ANUAL COMALFI, 29., 1999, Cartagena. Resumenes... Cartagena: COMALFI, 1999. p. 91. 